
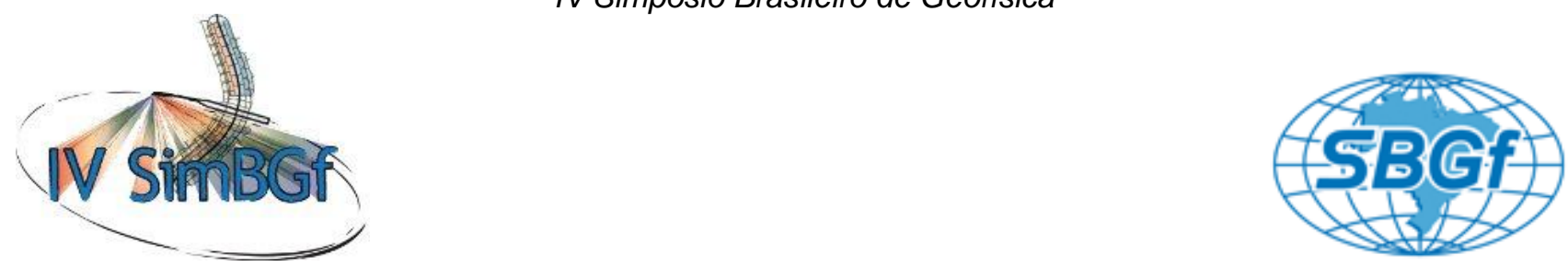

\title{
Parâmetros de fonte de sismos locais por inversão de formas de ondas
}

\author{
Ronnie Quintero ${ }^{1}$, Jiri Zahradnik², Lucas Vieira Barros ${ }^{3}$
}

1OVSICORI-UNA, Heredia, Costa Rica - rquniter@una.ac.cr

${ }^{2}$ Charles University, Prague, República Czech - jz@karel.troja.mff.cuni.cz

3 Observatório Sismológico - UnB, Brasília - DF, Brasil - Lucas@unb.br

Copyright 2010, SBGf - Sociedade Brasileira de Geofísica

Este texto foi preparado para a apresentação no IV Simpósio Brasileiro de Geofísica, Brasília, 14 a 17 de novembro de 2010. Seu conteúdo foi revisado pelo Comitê Técnico do IV SimBGf, mas não necessariamente representa a opinião da SBGf ou de seus associados. É proibida a reprodução total ou parcial deste material para propósitos comerciais sem prévia autorização da SBGf.

\section{Abstract}

The main purpose of this work is to invert seismic waveforms in order to obtain moment tensor for two medium size earthquakes detected by OVSICORI-UNA Costa Rica permanent seismic network, occurred in 2004, following the mainshock of November 20 at 08:07 UTC $(\mathrm{Mw}=6.4 \mathrm{GCMT})$. The inversion for the focal mechanism is made using the package ISOLA (Zahradnik et al., 2005, Sokos \& Zahradnik, 2008) and the seismicity are associated with a fault system that delineates the upper plate between the Caribbean plate and Panama Block. The obtained focal mechanisms are in agreement with results obtained by Pacheco et al. (2006) for this seismicity.

\section{Introdução}

O conhecimento preciso dos parâmetros sísmicos de fonte é pré-requisito para a compreensão de processos físicos que ocorrem durante os terremotos. Rotineiramente, são extraídas apenas da primeira chegada da onda $P$ importantes informações a cerca desses parâmetros, tais como: localização hipocentral, magnitude e solução de planos nodais. Entretanto, muito mais pode ser inferido a cerca de aspectos cinemáticos e dinâmicos das fontes sísmicas quando se analisa o sismograma em sua totalidade ou em determinadas faixas de freqüências. Normalmente, a utilização apenas da fase $P$ exige o registro do mesmo terremoto em várias estações. Já a análise mais detalhada das formas de onda de um evento pode reduzir substancialmente o número de estações necessárias à extração dos mesmos parâmetros. Neste trabalho é mostrado que o mecanismo focal de um sismo pode ser obtido, de forma confiável, usando-se informações contidas nas suas formas de ondas registradas por apenas quatro estações; o que não seria possível usando 0 método tradicional de polaridades. Particularmente utilizamos os registros de banda larga de duas réplicas do terremoto $(\mathrm{Mw}=6,4)$, ocorrido em 20/11/2004 na Costa Rica, às 08:07 (UTC).

A localização da seqüencia sísmica associada ao evento principal, de 20 de novembro de 2004, ocorrida no pacífico e na parte central da Costa Rica, foi realizada em outros estudos (Quintero et al., 2005, Pacheco et al., 2006) (Fig. 1). Neste trabalho nos limitamos a usar essas localizações para a inversão do tensor de momento sísmico de dois eventos específicos dessa sequência. Para informações sobre a tectônica da região consultar Pacheco et al.(2006), onde é dada uma lista completa de referências sobre o tema. A falha onde ocorreu esta sismicidade é parte de um sistema de falhas que marca o limite entre a placa do Caribe e a microplaca do Panamá, sistema de falhas conhecido como Cinturão deformado do Centro da Costa Rica. O sismo principal ocorreu em uma falha normal com uma pequena componente transcorrente e centróide localizado a $22 \mathrm{~km}$ de profundidade com réplicas distribuídas até a superficie (Fig. 1). O mecanismo GCMT (Global Centroid Moment Tensor) indica uma posível falha com orientação NW, com um mergulho de $77^{\circ}$ para NE. O evento foi sentido fortemente na zona epicentral, causando rupturas superficiais, liquefação local e danos em casas e edifícios pequenos. Teve uma intensidade (MM) VII em Parrita e Damas, (V-VI) em Jacó e Quepos, (IV-V) em Puntarenas e no Vale central do país (Alajuela, San Jose, Cartago; ver Fig. 1). A maioria dos danos ocorreu em Parrita e Damas, onde a estrada que une essas duas cidades foi fechada parcialmente. Também há relatos de danos na zona de Santos, localizada a $30 \mathrm{~km}$ a NE da zona epicentral (Observatório Vulcanológico e Sismológico OVSICORI da Universidade Nacional - UNA da Costa Rica < http:/www.ovsicori.una.ac.cr>).

Neste trabalho nos concentramos em mostrar a utilidade das formas de ondas obtidas por estações de banda larga pertencente à rede permanente do OVSICORI-UNA para a inversão do tensor de momento sísmico. Pacheco et al. (2006) fizeram inversões para o tensor de momento sísmico dos eventos aqui estudados. Entretanto, usaram dados telessísmicos e regionais de redes sísmicas temporais e outros métodos de inversão e de cálculo das 
funções de Green. Aqui usamos apenas dados locais de banda larga da rede permanente do OVSICORI -UNA. Mostramos como inverter para o tensor de momento sísmico, indicando os cuidados que se deve ter na preparação dos dados, especialmente no caso em que estes são obtidos automaticamente por sistema trigger, resultando, às vezes, em cortes abruptos, que podem causar problemas na hora da inversão. Também será discutido o caso em que aparecem nas formas de ondas, gravadas por instrumentos Guralp CMG-6TD, pulsos de período longo, impossibilitando o seu uso direto na inversão. Zahradnik e Plesinger (2005) discutiram esse problema para outros tipos de instrumentos.
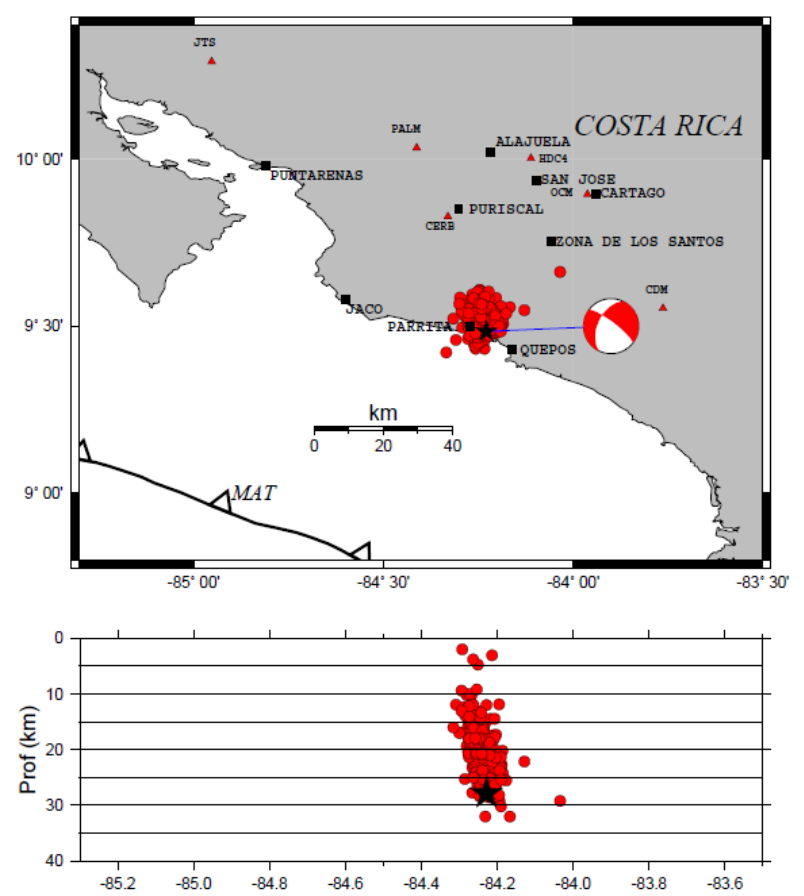

Fig.1. Sismicidade associada ao sismo de 20 de novembro de 2004 na Costa Rica (08:07 UTC), círculos vermelhos (sem distinção de magnitudes). Na parte inferior da figura está a distribuição dos sismos por profundidade. A estrela denota o sismo principal $(\mathrm{Mw}=6,4)$. As estações usadas na inversão estão indicadas por triângulos vermelhos e algumas cidades onde o sismo foi percebido são representadas por quadrados pretos. MAT denota a trincheira da América Central, encontro da placa de Cocos com a placa do Caribe e o bloco do Panamá. $\mathrm{O}$ beach ball indica o mecanismo focal GCMT ( http://www.globalcmt.org/).

\section{Dados e método de investigação}

A rede sismográfica do OVSICORI, pertencente à Universidade Nacional da Costa Rica (UNA), coleta e analisa rotineiramente dados sísmicos registrados na forma digital. A maioria das estações operou na forma analógica até 1992, quando começou a funcionar o sistema de registro digital, SEISLOG (Haskov e Utheim, 1992), com uma taxa de amostragem de $50 \mathrm{~Hz}$. Em 2003 entrou em operação o sistema de aquisição Earthworm, e a digitalização das estações analógicas passou a ser realizada no centro de registro do OVISICORI, localizado em Heredia, Costa Rica. Os sismômetros da rede são principalmente Ranger SS-1 (1 HZ), componente vertical. Paralelamente ao sistema Earthworm, a partir de março de 2010, o pacote ANTELOPE passou a ser usado na aquisição e procesamento de dados sismícos da rede do OVSICORI.

O evento de 20 de novembro de 2004 e suas réplicas foram registrados também por 5 estações Guralp, CMG6TD $(30 \mathrm{~s}-50 \mathrm{~Hz})$, com taxa $100 \mathrm{sps}$ (estações HDC4, PALM, OCM, CERB e CDM) e pela estação JTS, pertencente ao consórcio IRIS, administrada pelo OVSICORI. Esta estação conta com instrumentos Streckeisen STS-1 e STS-2 (120 s - $100 \mathrm{~Hz})$, com frequência de amostragem de 20 e $40 \mathrm{~Hz}$, respectivamente.

Todos os dados das estações são adquiridos pelo sistema Earthworm, usando-se um sistema de trigger. As localizações são feitas com o programa Hypocenter (Lienert, 1995) e a análise é feita com o pacote de análise de dados sísmicos SEISAN (Havskov e Ottemöller, 2008). Os dados das estações Guralp também são gravados na forma contínua, adquiridos por meio do sistema Scream. Os dados da estação JTS podem ser obtidos na forma contínua da página web do IRIS < http://www.iris.edu/hq/>. Os sismos estudados estão indicados na Tabela 1 e suas formas de ondas são apresentadas nas figuras 2 e 3 .

Inicialmente, os dados são transformados para o formato SAC ou GCF. Para realizar a inversão devemos ter os pólos e zeros da função de transferência dos sismógrafos, em radianos, na forma de velocidade.

Tabela 1. Parâmetros sísmicos dos eventos estudados neste trabalho, onde Prof=Profundidade e $\mathrm{M}_{\mathrm{d}}=$ Magnitude de duração.

\begin{tabular}{|l|l|l|l|l|l|}
\hline yyyymmdd & hhmmsec & Lat. & Long. & Prof. & $\mathrm{M}_{\mathrm{D}}$ \\
\hline 20041120 & 140855,3 & 9,574 & $-84,252$ & 15,1 & 2,9 \\
\hline 20041207 & 081515,2 & 9,11 & $-84,178$ & 21,0 & 4,5 \\
\hline
\end{tabular}

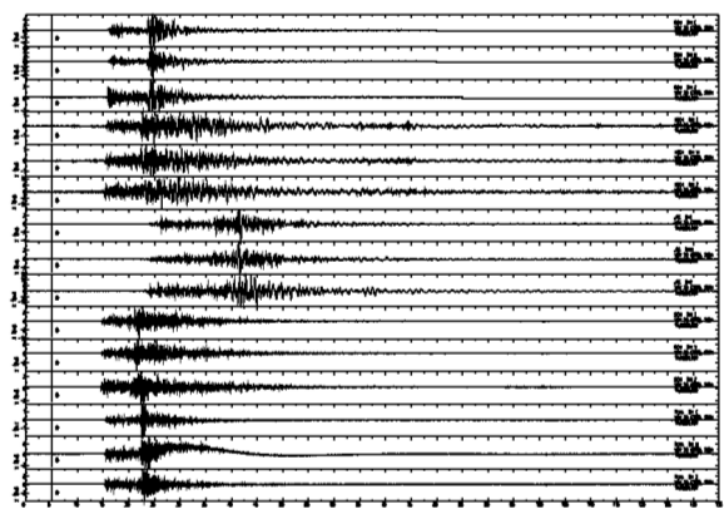

Fig. 2. Formas de ondas nas estações CDM, HDC4, JTS, OCM e PLMA do sismo de 20/11/2004, às 14:08:55,3 h (UTC), cujos dados foram usados neste estudo. Os dados da estação JTS se referem ao instrumento STS-1, adquiridos da página web do IRIS, já que o sistema de trigger usado com o Earthworm cortou o sismo em seu inicio. 


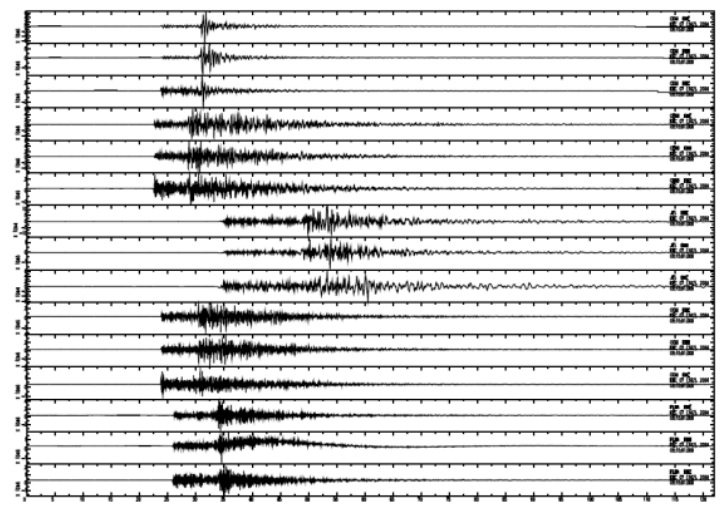

Fig. 3. Formas de ondas nas estações CDM, CERB, JTS, OCM e PLMA do sismo de 07/12/2004, às 08:15:15 h (UTC), cujos dados foram usados neste trabalho. A estação JTS se refere ao instrumento STS-1.

A inversão dos dados para obtenção do tensor de momento sísmico para esses dois eventos se realizou com o código ISOLA (Zahradnik et al., 2005, Sokos e Zahradnik, 2008) e o MATLAB. As funções de Green foram calculadas usando 0 modelo de velocidade unidimensional local determinado por Quintero e Kissling (2001). As funções de Green foram calculadas para um modelo de fonte pontual localizada acima e abaixo do hipocentro. Foram testados 10 hipocentros, 5 acima e 5 abaixo do hipocentro dado. Todos os registros são corrigidos pela resposta dos instrumentos, filtrados com um filtro banda passante e, finalmente, integrados para obter o deslocamento. Ao final se inverte por um mecanismo focal DC (double couple) e, ao ser encontrada a solução que produz a melhor correlação entre os sismogramas sintéticos e observados, em um dos 10 pontos testados, se realiza novas inversões, uma para cada fonte pontual situada sobre um plano que passa pelo hipocentro ótimo da solução anterior. Este plano é dividido em uma grade retangular, cuja separação depende das dimensões físicas da fonte investigada. O centróide (centro de gravidade da falha deslocada),corresponde à solução que produz o melhor ajuste entre os dados observados e sintéticos.

Durante a preparação dos dados, e especialmente ao se realizar a inversão, observou-se que existia pouca estabilidade nas soluções. Constatou-se que isto se devia à forma de onda da estação PLMA. Esta estação apresenta um pulso grande de período longo, o qual é observável facilmente na componente NS dos dados originais (figuras 4 e 5). Algumas vezes o pulso é difícil de ser observado. Porém, após a filtragem e a transformação dos dados de velocidade em deslocamento, é possível observar este problema, inclusive em outras componentes, em nosso caso, na componente EW da estação PLMA. Essas anomalias nas componentes foram estudadas para outros instrumentos e para outras zonas (Zahradnik \& Plesinger, 2005). Em nosso caso os dados de PLMA não foram usados na inversão, embora apareçam nas figuras com dados observados e sintéticos.
Existem casos em que as formas de ondas dos eventos obtidos por trigger apresentam rampas (veja Fig. 6) e ao passar esses dados de velocidade para deslocamento, obtivemos uma grande amplificação do ruído nas freqüências estudadas. Por esta razão se optou por cortar a forma de onda antes da rampa. Este corte deve ser automaticamente acompanhado por suavização (Sokos \& Zahradnik, 2008).

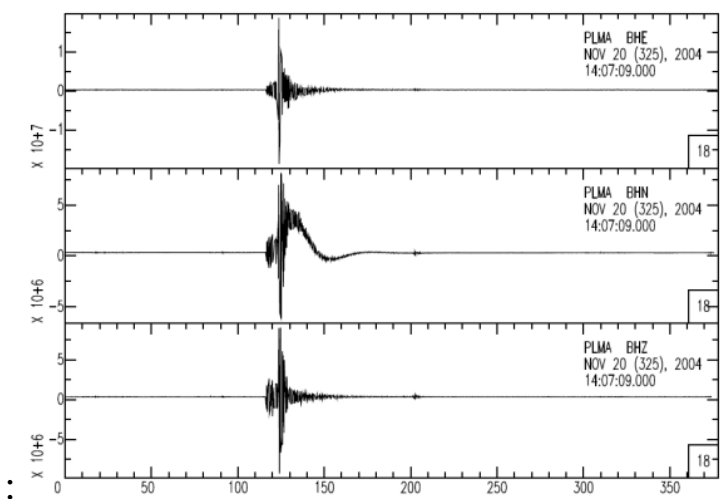

Fig. 4. Formas de ondas do sismo de 20/11/2004, às 14:08 h (UTC), registrado na estação PLMA, onde se observa o pulso de período longo na componente NS.

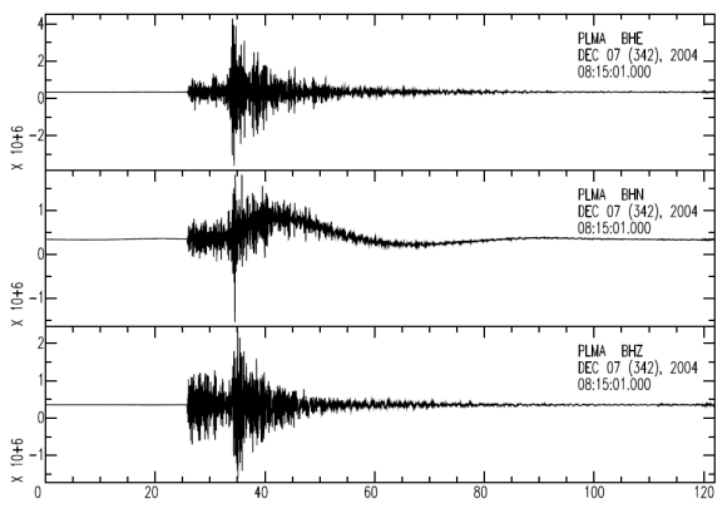

Fig. 5. Formas de onda do sismo do dia 7/12/2004, às $08: 15$ h (UTC), registrado pela estação PLMA. Novamente se observa, claramente um pulso de período longo na componente NS. 


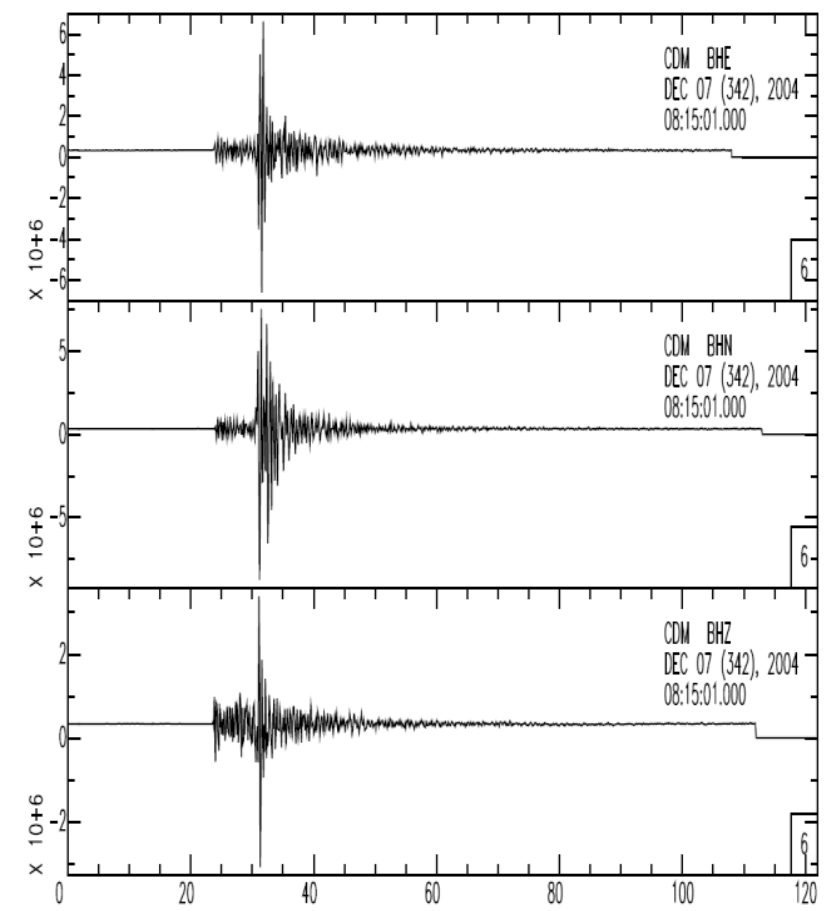

Fig. 6. Formas de ondas na estação CDM, do sismo de 07/12/2004, ocorrido às 08:15 h (UTC). Pode se observar uma forma de rampa nas três componentes, próximo de 117 segundos. Para usar os dados na inversão, estes foram cortados antes da rampa e depois suavisados.

\section{Resultados}

Os dois sismos estudados neste trabalho (20/11/2004, $2,9 \mathrm{~m}_{\mathrm{D}}$ e $07 / 12 / 2004,4,5 \mathrm{mD}$ ) foram registrados por instrumentos de banda larga de uma rede local do OVISICORI (CMG-6TD, $30 \mathrm{~s}-50 \mathrm{~Hz}$ ) e por uma estação IRIS (JTS), com instrumento Streckeisen (STS-1, $120 \mathrm{~s}$ $100 \mathrm{~Hz}$ ). Esses sismos são réplicas do terremoto de 20 de novembro de $2004(\mathrm{Mw}=6,4)$ e ocorreram em uma falha local, que é parte de um sistema de falhas do cinturão deformado do centro da Costa Rica. O tensor de momento de ambos os sismos indica uma falha com orientação NW-SE.

A inversão para o mecanismo $D C$ foi realizada com o código ISOLA (Zahradnik et al., 2005, Sokos e Zahradnik, 2008), que está baseado no método de Kikuchi e Kanamori (1991), modificado para eventos locais e regionais com o método do número de onda de Bouchon (1981). As funções de Green foram calculadas usando o modelo de velocidade unidimensional de Quintero e Kissling (2001). Todos os registros foram corrigidos pela resposta dos instrumentos, filtrados com um filtro bandapassante e, finalmente, integrados para obter 0 deslocamento. Foi usado um filtro caracterizado por quatro freqüências, $0,08,0,1,0,15$ e $0,2 \mathrm{~Hz}$, onde as freqüências extremas $(0,08$ e 0,2$)$ indicam a aplicação de uma janela do tipo cosseno, e $0,1-0,15$ denotam a faixa de frequência onde a resposta do instrumento é plana.
Outros filtros e modelos de velocidade foram testados. No entanto, os resultados com os parâmetros acima foram estáveis, como mostra a Fig. 7.

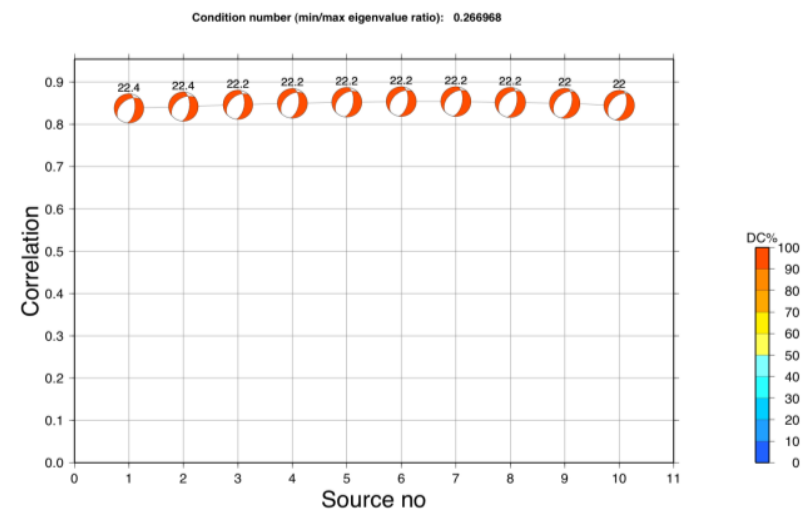

Fig. 7. Mecanismos focais obtidos para o sismo de 20/11/2004 para 10 fontes diferentes. Na vertical aparece a correlação entre as formas de ondas observadas e sintéticas. Para cada fonte sísmica (source no) se obtém um mecanismo focal e a correlação correspondente. Embora várias fontes tenham sido analisadas, aquí se mostra os resultados para 10 profundidades, começando por $13 \mathrm{~km}$ (source 1) e terminando em $22 \mathrm{~km}$ (source 10), com $1 \mathrm{~km}$ de espaçamento entre fontes. A maior correlação se dá para a fonte número 6 , que corresponde a 18 $\mathrm{km}$, que é a profundidade do centróide. A porcentagem DC é alta, 91,2\% (veja Fig. 8), indicando uma fonte sísmica simples, com ruptura singular.

Os mecanismos focais, obtidos para os dois sismos para um par de forças duplas estão na Tabela 2 e nas figuras 8 e 9.
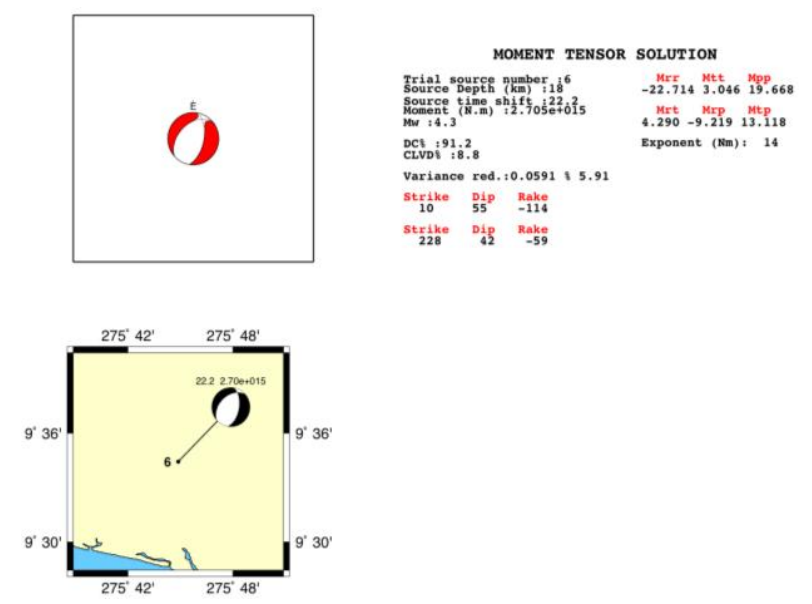

Fig. 8. Mecanismo focal obtido para o evento de 20 de novembro de 2004, às 14:08h (UTC). A melhor solução encontrada ocorreu para a fonte 6 , situada a uma profundidade de $18 \mathrm{~km}$. O mecanismo indica uma falha normal.

Tabela 2. Mecanismos focais obtidos para os dois sismos estudados neste trabalho.

\begin{tabular}{|l|l|l|l|l|l|l|l|l|}
\hline yymmdd & Time & Lat. & Long. & Prof & $\mathrm{Mw}$ & Strike & Dip & Rake \\
\hline 041120 & $14: 08$ & 9,57 & $-84,25$ & 18 & 4,3 & 228 & 42 & -59 \\
\hline 041207 & $08: 15$ & 9,11 & $-84,18$ & 22 & 4,1 & 16 & 65 & 18 \\
\hline
\end{tabular}



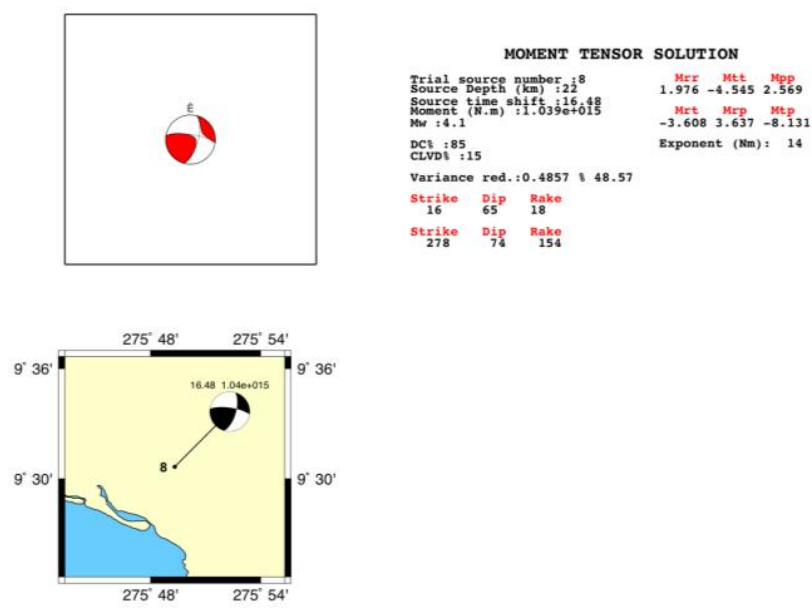

Fig. 9. Mecanismo focal obtido para o sismo de 7 de dezembro de 2004, às 08:15h (UTC). A melhor solução encontrada ocorreu para a fonte número 8 , localizada a $22 \mathrm{~km}$ de profundidade. $\mathrm{O}$ MF indica uma falha transcorrente, com pequena componente inversa.

Nas figuras 10 e 11 são mostrados os sismogramas calculados (vermelho) e observados (preto) para as estações analisadas neste trabalho.

Displacement (m). Inversion band (Hz) $\quad 0.080 .080 .120 .12$
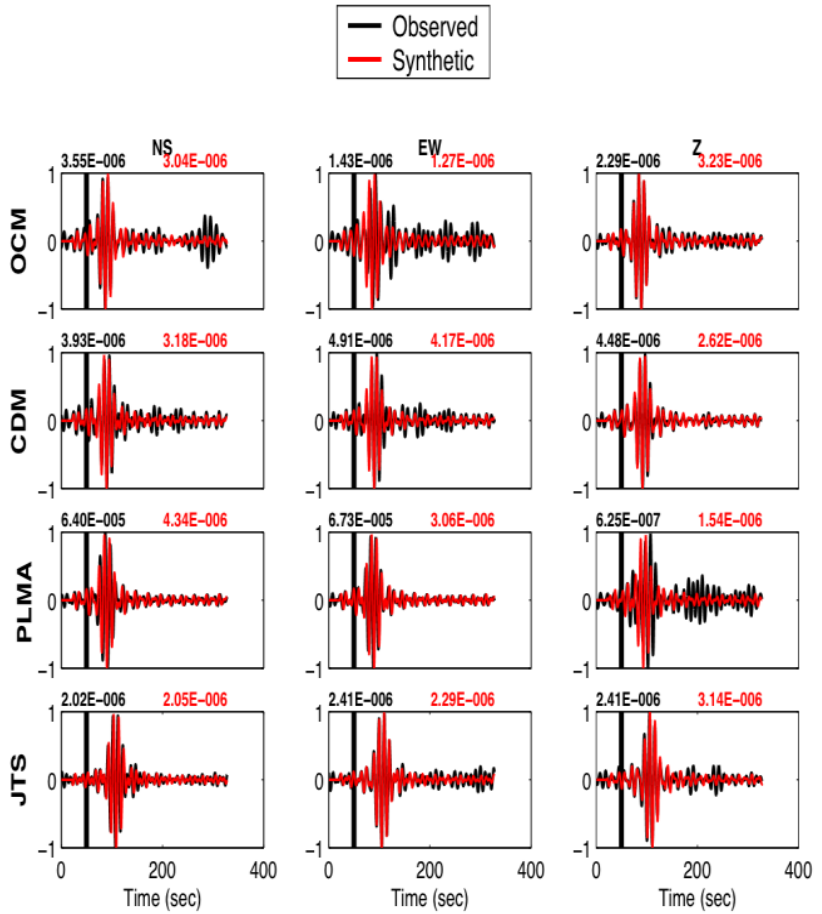

Fig. 10. Sismogramas sintéticos (vermelhos) e observados (pretos) das estações OCM, CDM, PLMA e JTS, para o sismo de 20 de novembro de 2004, às 14:08 h (UTC).
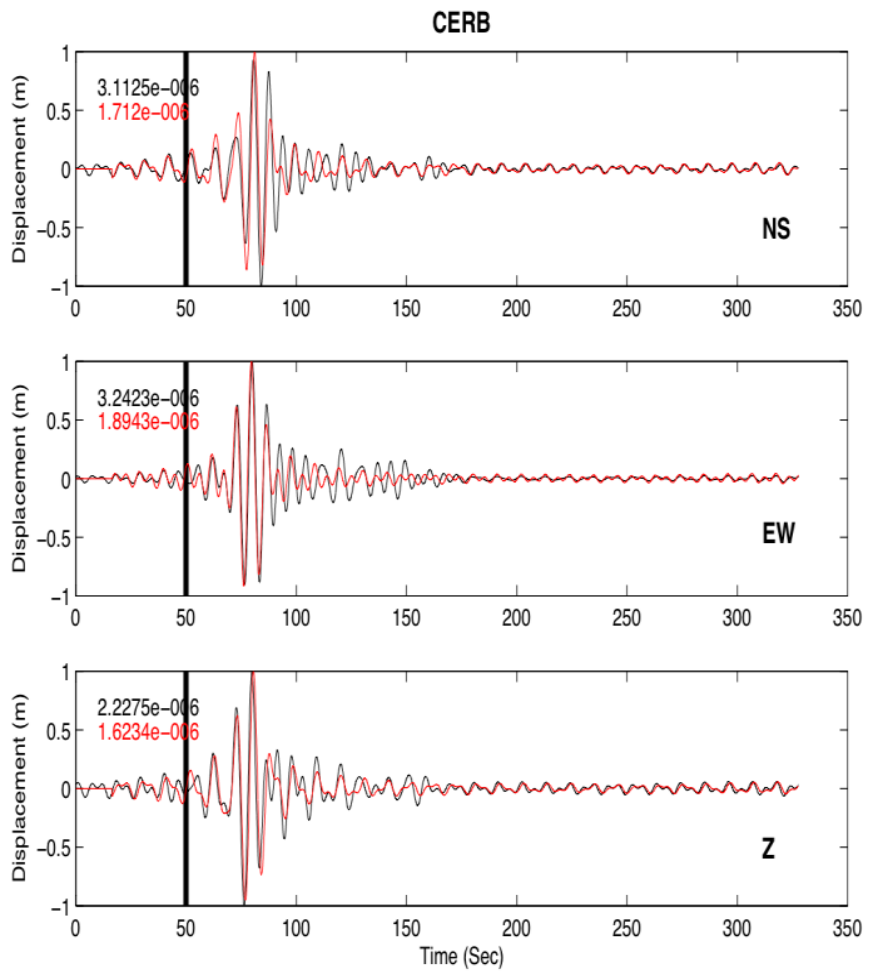

Fig. 11. Sismogramas sintéticos (vermelho) e observados (preto) para o sismo de 7 de dezembro de 2004, às 08:15 h (UTC), na estaçõn CERB.

\section{Discussão e conclusões}

Na região do Pacífico central da Costa Rica, em sua parte continental, tem sido detectado sismicidade desde 1984, quando foi instalada a rede do OVSICORI - UNA, e mais recentemente com a instalação dos instrumentos de banda larga, cujos registros foram usados na inversão de dois mecanismos focais de réplicas do terremoto de $20 / 11 / 2004(\mathrm{Mw}=6,4)$. Os resultados aqui obtidos são concordantes com os de Pacheco et al., 2006, obtidos para os mesmos sismos, usando o método de inversão de Randal et al. (1995) e os sismogramas sintéticos calculados com o algoritmo de Kennett (1983) e Randall (1994), usando o modelo de velocidade 1D de Quintero e Kissling (2001).

O mecanismo GCMT obtido para o evento principal de 20/11/2004 e os obtidos neste trabalho indicam que a sismicidade ocorreu numa falha transtensional localizada na frente do arco vulcânico da Costa Rica, e que diferentes tipos falhas se romperam após o sismo principal. Deduz-se também, que a fórmula de cálculo da magnitude de duração não está bem calibrada, produzindo, como foi o caso do evento 1 da Tabela 1 , magnitudes baixas.

Portanto, podemos concluir que a consistência dos resultados e a estabilidade das soluções indicam que o método de inversão aplicado neste trabalho, pode ser extendido para outras regiões, particularmente para a intraplaca brasileira, onde os sismos são raros e, geralmente, registrados por poucas estações. 


\section{Agradecimentos}

Agradecemos ao OVSICORI - UNA - Costa Rica, pela cessão dos dados sísmicos usados neste trabalho, e à Faculdade de Matemática e Física, da Universidade de Charles - Praga - República Checa, pelo apoio durante a visita de um dos autores (Ronnie Quintero), em dezembro 2006, período em que aprendeu a manusear o código ISOLA. As figuras foram geradas com o software GMT (Wessel and Smith, 1995).

\section{Bibliografia}

Bouchon, M., .1981. "A simple method to calculate Green's functions for elastic layered media”, Bull. Seis. Soc. Am., 71, 959-971.

Haskov, J., and Utheim, T.,1992. "SEISLOG and SEISAN: A complete system for seismic data acquisition and analysis", Cahier du Centre Europeen de Geodynamique et Seismologie, 5, 6774.

Havskov, J. \& Ottemöller, L. (Eds), 2008. SEISAN: The Earthquake Analysis Software, Version 8.2.1 Inst. of Solid Earth Physics, University of Bergen, Norway, $227 \mathrm{p}$.

Kennett, B. L. N., 1983. "Seismic Wave Propagation in Stratified Media", Cambridge University Press, Cambridge, U.K., $342 p$.

Kikuchi, M., and Kanamori,H., 1991. "Inversion of complex body waves III", Bull. Seis. Soc. Am. 81, 6, 2335-2350.

Lienert, B. R., and Havskov, J., 1995. "A Computer Program for Locating Earthquakes both Locally and Globally", Seism. Res. Let. 66 (5), 26-36.

Observatorio Vulcanológico y Sismológico de Costa Rica, Universidad Nacional, http:/www.ovsicori.una.ac.cr.

Pacheco, J., Quintero, R., Vega, F., Segura, J., Jiménez, W., González, V., "The Damas $(M w=6.4), 2006$. Costa Rica, earthquake of November 20, 2004; aftershocks and slip distribution", Bull. Seism. Soc. Am., 96, No 4, 12p.

Quintero, R., and E. Kissling, 2001. "An improved P-wave velocity reference model for Costa Rica", Geofís. Int., 40, 3-19.

Quintero, R., Vega, F., Segura, J., y Jiménez, W.,2004. "Estudio espacial del sismo $(M w=6.4)$ del 20 de Noviembre del 2004 en Costa Rica". Geofísica 61, 7-19.

Randall, G. E., 1994. "Efficient calculation of complete differential seismograms for laterally homogeneous earth models", Geophys. J. Int. 118, 245-254.

Randall, G. E., C. J. Ammon, and T. J. Owens, 1995. “ Moment tensor estimation using regional seismograms from a Tibetan plateau portable network deployment”, Geophys. Res. Lett. 22, 1665-1668.

Sokos, E., and Zahradnik, J., 2008. "ISOLA A Fortran code and a Matlab GUI to perform multiple point source inversion of seismic data". Computer and Geosciences.

Wessel, P., and Smith, W. H. F., 1995. "New version of the Generic Mapping Tools", released, EOS 76, 329.

Zahradnik, J., Serpetsidaki, A., Sokos, E, and Tseleis, GA.,2005. "Iterative deconvolution of regional waveforms and a double-event interpretation of the 2003 Lefkada earthquake, Greece". Bull. Seism. Soc. Am., 95, 159-172.

Zahradnik, J., and Plesinger, A., 2005. "Long-period pulses in broadband records of near earthquakes". Bull. Seism. Soc. Am., 95, 1928-1939. 